\title{
Evaluation of Student Work Using a Letter Grading Scheme in Design and Calculus
}

\author{
Sean Maw and Indy Lagu \\ Mount Royal University, Calgary, Alberta, Canada \\ seanmaw@shaw.ca and ilagu@mtroyal.ca
}

\begin{abstract}
This paper presents methods, pros and cons of using a letter grading system versus a percentage grading system, in engineering course component evaluations. In making evaluation criteria across deliverables qualitatively similar, letter grading shows performance equivalency across courses and subject areas, as well as departments, faculties and universities. What is worth an ' $A$ ' is not always a trivial discussion. But it is an easier discussion than what is worth $83 \%$ versus $85 \%$. How to letter grade various types of deliverables in a valid and equivalent fashion can still be challenging. But with thoughtful marking rubrics, many deliverables can be evaluated using letter grades. These can be combined to produce valid final letter grades. Overall, there are advantages to taking such an approach to evaluation, and these are discussed in the paper.
\end{abstract}

Keywords: design, calculus, grading, engineering

\section{INTRODUCTION}

Mount Royal University (MRU) recently implemented a change in the mapping from percentage grades to letter grades, making it easier to get A's and B's. In an effort to maintain consistency with letter grades of previous years, some instructors in the Engineering program attempted a novel subversion. They marked all Calculus and Design course components with letter grading, directly. This paper describes the lessons learned from this approach.

\section{IMPLEMENTATION}

For the Calculus course, every single question was marked using letter grades. Each question had a weighting. The final grade for a test was determined by summing the weighted GPA valuations for the letter grades. The meanings of the letter grades corresponded to MRU's Calendar i.e. A = Excellent, B = Very Good, C = Satisfactory, D = Minimal Pass, and F = Fail. At the end of the term, all evaluations were combined and normalized to a (GPA) score. That value was then translated back to a final course letter grade. Since an 'A' is very hard to achieve in this system, final grades were rounded up.

In the Engineering Design course, a very similar approach was used, with a few differences. A variety of types of assignments was used in this course, including a short-answer quiz, a "feasible solutions" assignment, two presentations, a recommendation report, two short essays, a log, a proposal memo and a participation mark. In each case, a rubric was provided to students that described what constituted an "excellent" performance for an A, a "very good" performance for a "B", etc. Aside from not applying a final grade bump at the end of term, the approach used was the same as for the Calculus course.

\section{DISCUSSION}

The authors/instructors noted several advantages in using the direct letter grading approach. It is more transparent than percentages. Dialogue shifts from the mark to the qualities of the work completed. As it is a more granular system ('A' versus 'B', as opposed to $68 \%$ versus $69 \%$ ), it is subject to less inconsistency and contention. Indeed, there is evidence in the literature suggesting that inter-rater reliability of letter grading is higher [1]. Students also seem to understand the meaning of their marks better. And the meaning of the marks is translatable across types of assignments, as well as across different disciplines of study. On the other hand, if one does not implement an "A+ $=4.3$ " grading level, then getting an ' $A$ ' is virtually impossible. There also tends to be some extra work for the instructor in developing different/careful rubrics for each type of assignment.

In terms of implementation tips, the authors found that one should avoid an even number of evenly weighted questions on an evaluation. This can lead to scores that fall exactly in the middle between two letter grades.

\section{CONCLUSIONS}

This informal "experiment" proved worthwhile and both authors felt that there was merit in piloting this grading scheme again in a range of different courses.

\section{References}

[1] Reznick, R.K., Colliver, J.A., Williams, R.G. and Folse, J.R. Reliability of different grading systems used in evaluating surgical students, The American Journal of Surgery, Vol. 157, No. 3, 1989, pp 346-49 